\title{
¿Habilidades naturales? Los procesos de trabajo en el caso de las trabajadoras de una fábrica química en Argentina' ${ }^{1}$
}

\author{
Natural abilities? Work processes in the case of the employees at a \\ chemical factory in argentina
}

Cecilia Anigstein ${ }^{2}$

\begin{abstract}
Resumen
Este trabajo explora, mediante una metodología cualitativa, los procesos sociales en los que se funda la subalternidad de las mujeres en el mundo del trabajo, en el caso de las obreras manuales no calificadas de un establecimiento productivo de la rama química, situado en el Gran Buenos Aires, Argentina. El abordaje incorpora aportes de la perspectiva de la relación social de sexos y examina el modo en que la construcción de la calificación técnica involucra la naturalización de las habilidades productivas de las mujeres en las líneas de montaje.

Nuestro propósito es contribuir a un conjunto de debates que indagan las especificidades de la experiencia social y del tipo de relación que las mujeres establecen con el trabajo en las sociedades latinoamericanas contemporáneas, en el caso de las operarias no calificadas de la industria.
\end{abstract}

Palabras clave: trabajo femenino - industria - procesos de trabajo - calificación - relación social de sexo.

\begin{abstract}
This research explores those social proceses in which women's subalternity at work is founded. By using a qualitative methodology. The case study were branche of chemical industrie placed in the Gran Buenos Aires, Argentina. This research approaches different perspectives such as the social relation between sexes, it considers the way by which the construction of technical qualification involves the naturalization of women's productive skills at the convey belt. Our purpose is to contribute to a set of debates about the particularlities of the social experience and the type of relation that women establish with their work, in the case of unskilled industry workers of contemporary Latin-American societies.
\end{abstract}

key words: woman work - industry - work processes - skills - relantionship sex.

Fecha de recepción: 19 de abril de 2012

Fecha de aprobación: 10 de abril de 2013

$1 \quad$ Este trabajo retoma una parte de los resultados de mi tesis de maestría (Programa de Posgrado en Ciencias Sociales UNGS-IDES), que versa sobre las intersecciones entre familia, trabajo y sindicalismo, para el caso de las operarias de dos fábricas de las ramas cosmética y química en Argentina.

2 Socióloga. Becaria Doctoral UNGS-CONICET (Argentina). ceciliaanigstein@gmail.com 


\section{INTRODUCCIÓN}

La reestructuración capitalista que tuvo lugar a escala global a partir de la década de 1970 trajo aparejada profundos cambios en los procesos productivos y en la morfología del trabajo colectivo asalariado. Supuso, entre otras cuestiones, un aumento de la participación de las mujeres en la actividad productiva. Sin embargo, paradójicamente la globalización creó "oportunidades de empleo para las mujeres", pero acompañadas por una flexibilización y precarización crecientes (Hirata, 2001).

En un contexto de generalización del uso flexible de la fuerza de trabajo, las trabajadoras de la industria se concentran en ramas de la producción cuya composición del capital se caracteriza por ser trabajo-intensiva (Antúnez, 2005; Carneiro Araújo, 2005). En estos sectores de la producción predominan la utilización de maquinaria obsoleta, los puestos de trabajo que comprenden tareas manuales repetitivas, la precariedad laboral y pésimas condiciones de trabajo en términos de impacto en la salud, acceso a la estabilidad laboral y a prestaciones sociales fundamentales.

Carneiro Araújo y Oliveira (2006) sostienen que las transformaciones de las matrices productivas durante el auge del neoliberalismo han tenido un indiscutible sesgo de género. El empleo de mujeres representa para muchas empresas fundamentalmente una alternativa para la reducción de costos. Asimismo, Helena Hirata (2010) advierte que las tesis de alcance universal que postulan la emergencia de un nuevo paradigma productivo alternativo y la "muerte del fordismo" han sido muy cuestionadas por distintas investigaciones empíricas que consideraron tanto la división sexual como la división internacional del trabajo. Estas contribuciones delatan la vigencia de modelos teóricos que parten de cierto arquetipo de trabajador: varón, calificado y polivalente. En este sentido, ponen al descubierto que la "especialización flexible" se aplica de manera diferencial según se trate de países industrializados o de países "en vías de industrialización", según se trate de mano de obra masculina o femenina. En otras palabras, que las tendencias hacia una mayor taylorización y su contrario, la tendencia hacia la no taylorización varía considerablemente cuando se consideran estas variables.

Por su parte, Marta Roldán (1992) argumenta que cada modelo de proceso de trabajo implica una distinta lógica o racionalidad empresarial, una determinada modalidad y un determinado nivel de calificaciones técnicas requeridas al plantel de producción. En efecto, la incorporación de mujeres a la industria manufacturera se asocia, por lo habitual y a nivel mundial, con la implementación del modelo de línea taylorista-fordista. Desde el punto de vista empresario, esto se debe a que existen diferencias entre los sexos preexistentes que tornan más redituable el empleo de mujeres en determinadas tareas: bajo costo laboral, superioridad productiva (el argumento de los dedos ágiles), presunta docilidad y paciencia. Por estos motivos, enfatiza Roldán, es pertinente una intervención feminista que conlleve el rechazo de una implantación directa de este debate en los términos exclusivos de la relación capital-trabajo. 
En efecto, siguiendo el hilo argumental de Roldán, en las representaciones y conceptualizaciones habituales sobre los sistemas tayloristas/fordistas y sus variedades post, se presupone la participación indistinta de varones y mujeres. La tipicidad de las formas organizativas analizadas se hace a base de un universo supuestamente neutral desde el punto de vista del sexo. En este universo neutral opera de modo exclusivo una lógica económica y/o política de clase, "no se investiga si esta lógica, al materializarse en una sociedad previamente jerarquizada por clivajes de género, no gesta, a su vez, variedades generizadas de un mismo sistema productivo" (Roldán, 1992: 109).

En este artículo nos propusimos indagar qué modalidades asume y cómo es experimentado el proceso de trabajo en el caso de las obreras no calificadas de la industria química ${ }^{3}$. La mirada se orientó hacia el lugar de trabajo, la fábrica, y se concentró en la labor cotidiana de las trabajadoras en las líneas de envasado desde la perspectiva de la relación social de sexos. El análisis ponderó el tratamiento de la "calificación" entendida como apreciación social del valor diferencial del trabajo en relación con la división sexual del trabajo. El presente trabajo sugiere que el carácter "no calificado" de las tareas femeninas en las líneas de producción podría ser interpretado como un efecto de naturalización de destrezas productivas no reconocidas, justamente por haber sido adquiridas en el ámbito doméstico familiar, como experiencias privadas e individuales.

Nos preguntamos: ¿Qué características asumen los procesos de trabajo de las operarias no calificadas del establecimiento seleccionado? ¿Cómo definen las trabajadoras sus destrezas productivas y su calificación profesional? ¿Qué saberes ponen en juego las operarias en el proceso de trabajo? ¿Por qué no son reconocidas socialmente? ¿Qué relación existe entre la división técnica y la división sexual del trabajo en los establecimientos fabriles?

En un primer apartado se presenta el enfoque de análisis, la perspectiva de la relación social de sexo y la división sexual del trabajo. A continuación se describen los procesos de trabajo en el establecimiento seleccionado. Finalmente, poniendo en juego las categorías de análisis presentadas, se analiza el carácter social de la construcción de la calificación desde la perspectiva de las trabajadoras.

\section{EL TRABAJO DESDE LA PERSPECTIVA DE LA RELACIÓN SOCIAL DE SEXOS}

Para pensar qué modalidad asumen los procesos de trabajo 4 de las operarias del establecimiento seleccionado hemos decidido apelar a la categoría relación social de

3 Para llevar adelante este estudio se utilizó un abordaje de tipo cualitativo. Se seleccionó un establecimiento de la rama química, que presenta una participación femenina de su fuerza laboral que supera ampliamente el promedio en la industria y en la rama, para el caso de Argentina, en la que las mujeres ocupan prioritariamente puestos considerados "sin calificación", taylorizados. Se entrevistaron trabajadoras vinculadas directamente a la empresa, en edad reproductiva y sindicalizadas. Asimismo, se entrevistaron dirigentes sindicales. El trabajo de campo para este caso se realizó entre octubre de 2010 y junio de 2011.

$4 \quad$ Sostenemos que el proceso de trabajo en las empresas capitalistas es fundamentalmente un proceso de valorización del capital. Ello es posible en tanto y en cuanto el trabajo se subordine a la lógica de acumulación en el proceso mismo de trabajo (Marx, 2002). Entendemos que los lugares de trabajo constituyen un terreno en disputa, donde 
sexos. El concepto de relación social de sexos fue utilizado inicialmente en la década de 1980 por las investigadoras francesas del GEDISST (Groupe de études sur la división sociales et sexuelle du travail), principalmente por D. Kergoat y se difundió luego en diversos países de América del Sur, como por ejemplo Brasil, de la mano de un conjunto nutrido de investigaciones sobre los procesos de reconversión tecnológica y organizacional de la producción y su impacto en las condiciones de trabajo de las mujeres en distintas ramas de la industria.

Esta categoría de análisis resulta fructífera por varias razones. Principalmente, habilita el debate con una perspectiva crítica más amplia de los estudios del trabajo (Harvey, 1998; Antúnez, 2005; Garza Toledo, 2001; entre otros) con notable impacto en Argentina en la última década, cuya contribución más destacada es haber restituido la envergadura de la noción de trabajo para el análisis social y, simultáneamente, haber puesto en debate los límites de las conceptualizaciones predominantes sobre el trabajo hacia fines del siglo XX5 . No obstante estos aportes, poco se ha avanzado -en Argentina en particular- en la articulación y el diálogo con el campo de los estudios de género, que recientemente ha experimentado un importante desarrollo, tanto a nivel local, como continental. Pero además, de acuerdo con García Castro (1992), la categoría de relación social de sexo permite considerar, a un tiempo y de manera explícita, el vínculo inexcusable entre dominación sexuada y dominación de clase, entre división social, técnica y sexual del trabajo.

El concepto de relación social de sexo tiene la particularidad de poner de relieve la dimensión material de la opresión de las mujeres: el trabajo. El trabajo, su organización y su división es uno de los principales objetivos y motivos de las contradictorias oposiciones que se desarrollan tanto entre las clases como entre los sexos, en el plano material y simbólico. Los conflictos que rodean al trabajo (mal pagado o sin retribución alguna) explican por qué el antagonismo parece ser el eje de las relaciones sociales de sexo, cuestión que no siempre queda clara cuando se utiliza el término género. Por otro lado, el hecho de tener en cuenta en esas relaciones la dimensión sexuada, pone

trabajadores/as y empresarios se enfrentan, cooperan, despliegan ofensivas o articulan respuestas, en el marco de relaciones verticales de dominación/subordinación entre el capital y el trabajo marcadamente asimétricas, por una parte, y de relaciones horizontales de competencia, entre los capitales entre sí (Gilly, 1985) e inclusive, entre los propios trabajadores/as. Históricamente, la generalización de la utilización de la maquinaria y el desarrollo de la gran industria impactaron profundamente sobre el colectivo obrero, provocando la utilización masiva de fuerza de trabajo femenino e infantil, la prolongación de la jornada laboral y la intensificación del trabajo. En rigor, la maquinaria constituyó el medio más poderoso para acrecentar la productividad del trabajo, para reducir el tiempo socialmente necesario para la producción de una mercancía. Paradójicamente, fue también el medio más poderoso para prolongar de forma efectiva la jornada de trabajo (Marx, 2002). Con la introducción de la línea de montaje, que surge como una de las novedades industriales en la primera posguerra, se abrieron nuevas posibilidades para la racionalización de la producción. La primera de ellas consistió en asegurar la circulación de piezas mientras los obreros permanecen quietos en sus puestos de trabajo. Con esto se obtuvo una ventaja doble: se economizó en mano de obra auxiliar y se logró una regulación mecánica -autoritaria- de la cadencia del trabajo. Una segunda posibilidad se relacionó con el acceso de las mujeres a puestos antes exclusivamente masculinos (Coriat, 2008).

5 En Argentina, en esta perspectiva se inscriben las investigaciones de Abal Medina, Paula; Wyczykier, Gabriela; Diana Menéndez, Nicolás; Battistini, Osvaldo, entre otros investigadores, como también así diversas publicaciones del Taller de Estudios Laborales (TEL), todas ellas referenciadas en la bibliografía. 
en cuestión el conjunto de las otras categorías sociológicas que se dan como evidentes, en primer lugar el concepto mismo de clase social y el de trabajo. Además, este concepto permite examinar las estrechas interacciones entre las relaciones de sexo y las relaciones de clase y no se limita a estudiarlas de forma paralela (Pfefferkorn, 2007).

Pfefferkorn (2007), señala que la resistencia a la hora de aceptar el concepto de relación social de sexos está ligada a la desaparición de los análisis en términos de clase social en países como Inglaterra o Francia desde fines de los años setenta. A lo largo de los ochenta y noventa, el eclipse de los estudios en términos de clase junto al predominio internacional de la lengua inglesa facilitó la difusión y éxito del término género y mermó la suerte de su homónimo, que recuperamos en este escrito. No obstante, debe recalcarse que las contribuciones empíricas y teóricas de autoras como Joan Scott, Bárbara Weinstein, Heidi Hartman, Temma Kaplan, Elizabeth Hutchison, entre otras, no se ajustan a los términos de esta argumentación. Por lo tanto, merecen ser consideradas en profundidad las líneas de disonancia y coincidencia respecto de la perspectiva propuesta. Dicho ejercicio excede los objetivos propuestos en este trabajo.

La noción de relación social de sexo guarda una estrecha relación con la de división sexual del trabajo. A continuación nos detenemos en estas dos definiciones para luego concentrarnos en el modo en que este enfoque impacta en la noción de calificación.

Para Kergoat (2003), toda relación social debe ser entendida como una tensión que recorre la sociedad. Tensión que cristaliza en retos en torno a los cuales los seres humanos se enfrentan para producir sociedad o para reproducirla. A su vez, las relaciones sociales son múltiples y ninguna de ellas determina la totalidad del campo que estructuran: "juntas tejen una trama e impulsan su dinámica, son consustanciales" (Kergoat, 2003: 845). Son consustanciales en la medida en que resulta imposible desenmarañar las relaciones sociales que componen la matriz societal. En todo caso, es el investigador quien debe "separar" las relaciones sociales. A partir de esta definición general de relación social es posible explicar las especificidades de la relación social de sexo.

La relación social de sexo es de la misma índole que el resto de las relaciones sociales, aunque posee características propias: está presente en la totalidad del espacio-tiempo conocidos, pero asume formas singulares. Implica la construcción de grupos sexuados (no productos de destinos biológicos sino constructos sociales) por tensión, oposición o antagonismo en torno a un reto, el reto del trabajo. El trabajo es la base material de la relación social de sexo y el naturalismo es la ideología de su legitimación, la "doxa del sexo". Se basa ante todo en una relación jerárquica, una relación de poder, y no un mero principio de clasificación.

Cabe destacar que no se trata del trabajo entendido en un sentido acotado, como trabajo asalariado, sino del trabajo como producción del vivir 6 , en una doble acepción:

$\overline{6}$ Hirata, H. y Zariffian, P. (2007) redefinen el concepto de trabajo como producción del vivir. Esta categoría intenta reponer en la definición de trabajo su carácter sexuado, por un lado, así como la distinción e historización de 
en el plano colectivo incluye el trabajo asalariado o no, mercantil o no, formal o informal, trabajo doméstico, de cuidados corporales y afectivos; y en el plano individual, ya que mediante el trabajo el individuo transforma su entorno y es transformado, produce y se produce a sí mismo, lo que obliga a tomar en cuenta la subjetividad.

Ahora bien, el enfoque del sexo como relación social se nutre de los debates en torno a la noción de división sexual del trabajo. Las reflexiones en torno a la división sexual del trabajo se inician en la década de 1970 bajo el impulso del movimiento feminista, momento en que proliferan un conjunto de trabajos desde las ciencias sociales que asientan las bases teóricas del concepto. Una de las primeras tesis que surgen de dichas conceptualizaciones es aquella que encuentra la base de la opresión en la enorme masa de trabajo invisible realizado sin remuneración por las mujeres, que tiene dos particularidades esenciales: no es un trabajo para ellas mismas, sino para otros y se realiza en nombre de la naturaleza, el amor y el deber materno?.

Para Daniele Kergoat y Helena Hirata (2007), la división sexual del trabajo surge de una forma específica de relación social: es la forma de división del trabajo que surge de la relación social de sexo y, simultáneamente, es un factor determinante para la sobrevivencia de dicha relación. Tiene como característica la designación prioritaria de los varones en la esfera productiva y de las mujeres en la esfera reproductiva. Y, simultáneamente, la apropiación de los varones de las funciones con mayor valor social: políticas, religiosas, militares, entre otras.

De acuerdo con estas autoras, la especificidad del concepto de división sexual del trabajo está fundada en dos principios organizadores: un principio de separación -existen trabajos de hombres y trabajos de mujeres- y un principio jerárquico -los trabajos de hombres valen más que los trabajos de mujeres-. Estos principios son válidos para todas las sociedades conocidas en tiempo y espacio y son aplicados mediante un proceso específico de legitimación: la ideología naturalista, que reduce

las dos vertientes de la definición de trabajo elaborada por la economía política clásica: la que se refiere a las relaciones de los seres humanos con la naturaleza y la que se refiere a las relaciones que se establecen entre los seres humanos.

$7 \quad$ En el marco de estos debates, las historiadoras Heidi Hartman (1980) y Joan Scott (1993) destacaron la presencia femenina en la actividad económica remunerada en el período de transición al capitalismo, tanto en Europa occidental como en Estados Unidos. Ambas autoras enfatizaron el impacto que tuvo la legislación protectora del trabajo femenino e infantil en la profundización de la división sexual del trabajo durante el siglo XIX y principios del XX. Hartman acudió a la noción de patriarcado para explicar de qué modo la organización jerárquica de la familia, precedente al advenimiento del capitalismo, se articuló con la producción industrial. Los empresarios aprovecharon muchas veces estas estructuras para reducir costos y/o obtener mayores beneficios. Eventualmente, encontraron en los trabajadores hombres aliados para impulsar la segregación laboral por sexo, que les permitió abonar remuneraciones muy inferiores a las mujeres. Scott, en cambio, afirmó que el proceso de industrialización no modificó radicalmente el modo en que las mujeres de desenvolvían en el hogar, la domesticidad y el trabajo remunerado fuera de la casa. De este modo, criticó las posiciones tributarias de la obra de Federico Engels para las cuales la industrialización provocó la separación del hogar y el trabajo, y obligó a las mujeres a elegir entre domesticidad o salario. Scott sitúa la génesis de la división sexual del trabajo, así como se cristalizó a inicios del siglo XX, en un giro discursivo en torno a la figura de la mujer trabajadora que tuvo lugar en el siglo XIX y que inspiró buena parte de la legislación protectora del trabajo femenino. Este modo de distribución sexual del trabajo, que asumió un carácter normativo, se constituyó en la base sobre la cual se diseñó la legislación laboral del capitalismo industrial. 
el género al sexo biológico, y las prácticas sociales a papeles sociales sexuales que se remiten y fundan en un destino natural de la especie. Lejos de ser una relación social inmutable, ostenta una enorme plasticidad y sus expresiones concretas varían en el tiempo y el espacio, así como ha sido extensamente documentado por la antropología y la historia.

Ahora bien, como ya se señaló, los procesos de trabajo interpelados desde esta perspectiva habilitan la problematización de la noción de "calificación" utilizada en los estudios del trabajo. Este escrito se propone ejercitar dicha problematización para el caso de las operarias de una planta de producción de la industria química.

\section{LAS OPERARIAS EN LAS LÍNEAS DE PRODUCCIÓN. EL CASO DE ANAERÓBICOS SRL}

Anaeróbicos SRL es una empresa dedicada a la producción de cianoacrilatos, trabas anaeróbicas para piezas mecánicas, aceites lubricantes penetrantes para uso industrial y adhesivos y selladores para uso doméstico. Además se dedica a la comercialización de distintos productos adhesivos, marcadores y de mantenimiento automotriz e industrial importados.

En Argentina cuenta con dos plantas. Una de ellas, ubicada en la provincia de San Luis, cuenta con alrededor de 15 trabajadores varones ${ }^{8}$ y se dedica principalmente a la elaboración y procesamiento de las materias primas. La segunda planta, situada en la ciudad de San Martín, provincia de Buenos Aires, cuenta con casi 100 trabajadores (48 de estos son mujeres). Allí se producen tubos colapsables de aluminio, se fraccionan y envasan las materias primas, se almacena y distribuye la producción. Esta empresa comercializa gran parte de sus productos mediante marcas de terceros y/o a granel. De acuerdo con lo indicado en su sitio web oficial, Anaeróbicos S.A., exporta el 50\% de su producción, principalmente en el ámbito del MERCOSUR, pero también al resto de América Latina, Estados Unidos, Europa y Asia.

Anaeróbicos inició sus actividades en 1972, siendo una pequeña empresa familiar de capitales nacionales. En diciembre de 2007 fue adquirida por la transnacional de origen norteamericano Illinois Tool Works Inc. (ITW) e integra el grupo ITW Performance Polymers \& Fluids, con presencia en cincuenta y dos países. Los testimonios coinciden en que el cambio de dueños implicó modificaciones en la organización del trabajo en la planta: la incorporación de nuevas máquinas que requieren menos puestos de trabajo, la masculinización de algunos puestos, cambios en las medidas de seguridad y el control de calidad y, especialmente, "mucha presión con el tema de la productividad". Asimismo, la nueva firma ha realizado actividades de capacitación para los maquinistas (técnicos mecánicos, exclusivamente varones), de las que han sido excluidas las operarias por pertenecer a las categorías profesionales inferiores. Es de destacar que los cambios introducidos no significaron la automatización del proceso productivo,

8 De acuerdo con el testimonio de una delegada de la planta de San Martín. 
sino fundamentalmente un aumento de la intensidad del trabajo y la introducción de mecanismos de disciplinamiento laboral que redundaron, básicamente, en mayor rentabilidad empresarial.

La planta se encuentra compuesta por tres sectores: Línea o Envasado (donde se fraccionan y envasan los productos), TUBEX (donde se fabrican los tubos de aluminio) y Depósito. En el depósito se desempeñan ocho trabajadores varones por turno, cuya tarea consiste en armar los pedidos y despacharlos a los fletes. El sector de Envasado se encuentra a su vez subdividido en distintas áreas: el sector de las bateas y envasado (donde se fraccionan y envasan parte de los productos); el sector de blíster donde con máquinas embaladoras se envasan los productos en pomos y frascos y luego se estuchan (se colocan adentro de cajas). Por último, se encuentra la cabina de Siano (compartimiento aislado del resto de las máquinas, completamente vidriado, donde se desempeñan dos operarios) donde se envasan adhesivos instantáneos que requieren refrigeración permanente. En los dos primeros subsectores del envasado se desempeña personal femenino. No ocurre lo mismo con la cabina de Siano. Salvo en casos excepcionales, las operarias ocupan alternativamente los distintos puestos de su sector, de acuerdo con la planificación diaria de la producción.

La masculinización de los puestos de la cabina de Siano es reciente. Una de las delegadas sindicales gremiales entrevistadas se desempeñó junto a otra compañera durante cuatro años en la cabina de Siano. Ambas fueron automáticamente removidas de esos puestos cuando ella comenzó a ejercer la representación gremial del establecimiento, en 2007. Según relata, la incorporación de varones en esos puestos implicó una categorización salarial superior de los mismos y la adición de nuevas tareas auxiliares.

En el sector TUBEX también se incorporaron varones en puestos tradicionalmente ocupados por mujeres, aunque sin relación alguna con la composición de la comisión interna, lo que resulta un indicio acerca de la índole de la masculinización de algunos puestos como una política empresarial que, según se desprende de los testimonios, se originó con el cambio de dueños a fines de 2007. Este sector, en el que se elaboran tubos colapsables de aluminio, cuenta con tres líneas de producción donde se desempeñan alrededor de doce operarias por turno, acompañadas, en cada una de las líneas, por un maquinista (técnico, varón, de categoría profesional superior). En TUBEX las trabajadoras están expuestas a mayores riesgos químicos debido a que no pueden realizar correctamente su trabajo si utilizan los elementos de seguridad personal requeridos (principalmente guantes y protectores de ojos), lo que ha ocasionado que muchas de ellas padezcan enfermedades en la piel, la más frecuente es la dermatitis.

En general, imprudencias como no usar los elementos de seguridad reglamentarios guardan una estrecha relación con la presión que ejercen sus superiores para alcanzar los objetivos de productividad. Las características que asumen los procesos de trabajo en la línea y la proliferación de "conductas imprudentes"-en rigor, responder frenéticamente a la presión de los superiores- multiplican los riesgos a los que se encuentran 
expuestas las trabajadoras ${ }^{9}$. En suma, el carácter repetitivo de las tareas realizadas, las posturas corporales requeridas y, especialmente, la exposición permanente a riesgos químicos impactan negativamente en la salud de las operarias. En consecuencia, las trabajadoras padecen enfermedades y malestares, sobresale por su frecuencia la dermatitis. Asimismo, la presión permanente para alcanzar los objetivos de productividad multiplica los riesgos de accidentes; mientras que el sistema de turnos rotativos significa un gran obstáculo para el desarrollo personal y la organización familiar y contribuye al deterioro global de la salud de las trabajadoras. Luego de varios años de labor en estas empresas, el desgaste físico, el deterioro de la salud y el sufrimiento psíquico experimentado, afectan profundamente la vida cotidiana de estas mujeres y ello cobra visibilidad de una manera dramática en el ámbito familiar.

\section{LA CALIFICACIÓN DE LAS TAREAS EN LA LÍNEA}

Cuando en situaciones de entrevista se les preguntó a las trabajadoras por los saberes requeridos y por la calificación del trabajo en la línea, en todos los casos ellas aseguraron que su tarea no es calificada. Simultáneamente, cuando se les preguntó por qué creían que la mayoría de los trabajadores de su establecimiento que ocupaban puestos en las líneas de envasado eran mujeres, invariablemente y sin rodeos respondieron que es un trabajo de mujeres. Ahora bien: ¿Cómo definen las trabajadoras sus saberes productivos? ¿Qué relación existe entre la división técnica y la división sexual del trabajo en el caso analizado?

A partir de la década de 1980 tuvo lugar un profundo debate en torno a la calificación y el saber-hacer en el trabajo frente al fenómeno de la flexibilización que daba sus primeros pasos en aquellos años. En ese contexto, fue cada vez más frecuente en la sociología laboral el reemplazo de las nociones de clase obrera y de calificación por las de cohesión social y especialmente por la noción de competencias (Alaluf, 2003).

Sin embargo, estudios críticos pusieron en evidencia los problemas de los nuevos enfoques y revalorizaron la vigencia de aportes conceptuales en apariencia envejecidos. Para Marcelle Stroobants (1999), con el auge de las ideas sobre la flexibilidad laboral y nuevas modalidades de organización del trabajo "el mito de la fábrica sin hombres fue reemplazado por el mito de la fábrica sin fallas". El hecho de que el taylorismo continúe siendo el paso obligado de todas las reflexiones sobre las denominadas nuevas formas de organización del trabajo, ya sea en clave de ruptura (postaylorismo) o de continuidad (neotaylorismo) pone de relieve que las afirmaciones acerca de los cambios en los paradigmas tecnológicos y de los modelos de organización de las empresas merecen, al menos, ser sometidas a algunas preguntas: ¿es un asunto de teoría o un discurso social? ¿El cambio de paradigma procede de los investigadores o de la empresa?

Desde 2007 la empresa ha colocado en la entrada del establecimiento, donde fichan los trabajadores un panel contador de accidentes que cada 160 días sin accidentes vuelve a cero. Esta política de prevención de accidentes contrasta con el día a día narrado por las operarias. Un ejemplo de ello es la ausencia de una enfermería en la planta química: "Son los supervisores los que diagnostican. Hay un botiquín. Tenés para el dolor de cabeza, dolor de estómago, algún antiinflacionario, algo para los ojos" (delegada de 31 años. San Martín, marzo de 2011). 
Para responder estas preguntas Stroobants apela a la definición de calificación de Naville de fines de los años cincuenta. Para este, la calificación debe ser entendida fundamentalmente como una apreciación social del valor diferencial del trabajo. Esto significa que surge de un acto de clasificación social y en tanto cualquier acto de clasificación comporta necesariamente una parte de arbitrariedad, buscar en los contenidos del trabajo determinantes de la calificación es querer comparar incomparables.

Para explicarlo con mayor claridad, Stroobants realiza un recorrido por los estudios sobre el conocimiento de los trabajadores y encuentra que en ese campo los términos saber y hacer aparecen con mucha frecuencia juntos. La autora encuentra además que la expresión saber-hacer sirve como recurso para reconstruir de manera solapada las oposiciones características entre categorías de conocimiento: intelectual-manual, abstracto-concreto, formal-informal. En última instancia, la expresión saber-hacer es utilizada por los enfoques empiristas para saldar la ambigüedad propia que surge de la espontaneidad en la descripción de los procesos de trabajo. Como resultado, pareciera que el saber-hacer constituye el contenido de la calificación. Tal es así que muchos estudios se dedican a identificar los desfasajes entre saberes adquiridos y saberes requeridos; entre el trabajo prescrito en una calificación y el trabajo real que la misma comporta; entre calificación oficial y calificación efectiva. Estas cuestiones, enfatiza Stroobants, más que las conclusiones de un análisis deben considerarse un punto de partida, ya que justamente ese desfasaje es constitutivo del proceso mismo de calificación.

En otros términos, en ausencia de un contenido objetivo, la calificación ya no aparece como una cosa sino como lo que está en juego en estrategias de los actores sociales. En última instancia, expresa una determinada correlación de fuerzas entre los actores de la producción. Por eso, más que comprender los mecanismos técnicos que se corresponden con una determinada calificación, la sociología debe orientarse a la investigación de los procesos de construcción social de dicha calificación.

Ahora bien, anteriormente afirmamos que las operarias químicas consideran que su trabajo no es calificado. Luego afirmamos que las operarias están convencidas que su trabajo es un trabajo para mujeres. Tomando en consideración la definición de calificación propuesta por Stroobants como apreciación social del valor diferencial del trabajo y como producto de una acción clasificatoria, nos preguntamos ¿Qué relación puede establecerse entre la "no calificación" de sus tareas y su condición femenina?

Marta Roldán (1992) alertó sobre la centralidad que adquiere la temática de las calificaciones, debido a que las jerarquías de género en los procesos de trabajo se construyen fundamentalmente, aunque no exclusivamente, basadas en las dimensiones socioeconómicas y simbólicas de la calificación. Para Mary García Castro (1992) la preferencia por el uso de fuerza de trabajo femenina en sectores en los cuales predominan los requerimientos relacionados con la minuciosidad, la agudeza visual o la motricidad fina, pone de manifiesto la estrecha relación existente entre división técnica, división social y división sexual del trabajo. Esta vinculación estaría explicando 
la índole de la segmentación en determinadas ramas de la producción industrial y su encasillamiento en puestos "sin calificación".

En una entrevista con el secretario de organización del Sindicato del Personal de Industrias Químicas y Petroquímicas (SPIQyP) se obtuvieron las siguientes apreciaciones sobre las mujeres que trabajan en el sector químico:

"¿Qué tipo de tareas hacen las mujeres?

En la línea de producción. Trabajan al ritmo de, por ejemplo, el envasado, etiquetado, poner tapa de botella. Y son trabajos que no requieren gran calificación. Porque generalmente, como el trabajo es artesanal, requiere más prolijidad; y la mujer en ese sentido es más prolija que el hombre. Y tal vez que son menos combativas, reclaman menos, todo ese tipo de cosas, eso va a depender de nosotros, en la medida que hagamos madurar el factor subjetivo en las mujeres como decíamos" (secretario de organización del SPIQyP, Buenos Aires, en noviembre de 2010).

Los testimonios de las operarias parecen ir en el mismo sentido. Como ya se señaló, las trabajadoras definieron su puesto de trabajo como femenino. Frente al pedido de justificación sobre dicha afirmación, obtuve respuestas así como: "Porque el trabajo nuestro es más específico", "es como un trabajo fino"; "Somos más detallistas"; "Las mujeres somos más rápidas en los trabajos manuales que los muchachos".

A partir de los relatos recogidos se advirtió que las operarias asocian sus habilidades con cualidades naturales, que son definidas como femeninas en tanto se diferencian de otras cualidades "masculinas". La velocidad de los movimientos manuales y el tamaño corporal o la paciencia son puestas en el mismo plano. Esto puede observarse cuando definen su labor y lo comparan con el trabajo de los operarios varones.

"El trabajo es más de mujer que de hombre

¿Por qué es más de mujer?

Y porque las mujeres somos más rápidas para sacar los pomos que los varones aparte que los varones tienen los dedos más grandes y los picos son muy chiquititos, puede ser por eso. A los varones por ahíse le pasan más pomos fallados que a las mujeres. Y otra cosa es que tienen menos paciencia también, en las máquinas, donde yo estoy por lo menos" (operaria de 28 años. San Martín, mayo de 2011).

Estas afirmaciones no se refieren solo a la experiencia cotidiana, surgen además de la "puesta a prueba" de las habilidades de los operarios varones por parte de las mujeres, tal como puede observarse en el siguiente extracto de entrevista:

"Pero los hombres no son tan capaces con las manos como las mujeres. Con la gricera, por ejemplo, con una máquina que se llama automática y otra máquina que se llama F8 donde se colocan picos y eso lo hemos comprobado porque hemos puesto compañeros a prueba a ver si podían tener la misma habilidad que las mujeres por el tema de que van dos cartuchos y tenés que poner dentro de unos papelitos lo picosy después a los costaditos, si no tiene la misma habilidad que tiene una mujer, $\mathrm{mmm}$, 
en sí, eso de que no se vaya a pasar un pico" (operaria y delegada de 36 años. San Martín, mayo de 2011).

En cambio, cuando las trabajadoras se refieren a las tareas "técnicas", no dudan en definirlas como masculinas:
"¿Y el trabajo técnico, mecánico?
Son los muchachos que están
¿Por qué sí los muchachos y no las mujeres?
Porque creo que de mecánica no somos muchas las que sabemos, mecánica: estar con grasa, tornillos" (operaria y delegada de 36 años. San Martín, mayo de 2011).

Sin embargo, los relatos también delatan que las trabajadoras tienen saberes que ponen en juego en el proceso de trabajo aunque los mismos sean decodificados por ellas y su entorno como atributos naturales de las mujeres. Recordemos la definición de calificación como apreciación social del valor diferencial del trabajo, que surge de un acto de clasificación social, que por esa razón comporta una parte de arbitrariedad y, en última instancia, expresa una correlación de fuerzas. Por lo tanto, las calificaciones no están determinadas exclusivamente por los contenidos concretos que asumen los trabajos.

A pesar de todo lo expuesto, hemos hallado elementos que horadan las representaciones naturalizadas de las operarias sobre sus saberes productivos: ellas tienen conocimientos técnicos, los ponen en práctica en su trabajo cotidiano y consideran que no son reconocidos por sus superiores. Dichos elementos nos permitieron problematizar el destino "natural" de las mujeres en líneas de producción, realizando tareas manuales con objetos pequeños y a altas velocidades:

\begin{abstract}
"En las máquinas con las que ustedes trabajan, si de repente alguna funciona mal o tiene algún problema ¿lo pueden resolver ustedes o normalmente tienen que acudir a alguien?

Y en el caso del sector de nosotras hay más de una chica que sí sabe cómo se desarma, cómo se puede limpiary lo hacemos. Pasa que antiguamente se acostumbraba eso y ahora no, porque tenemos mecánicos y hay cosas que las chicas no la hacen" (operaria de 28 años. San Martín, mayo de 2011).
\end{abstract}

Pero los conocimientos técnicos de las operarias no son reconocidos por sus pares varones, ni por sus superiores, independientemente que el convenio colectivo de trabajo de la rama química contenga una cláusula que prescribe la igualdad de géneros: Igual trabajo, igual salario ${ }^{10}$. En el marco de una entrevista, una delegada sindical de la planta hizo referencia al modo en el cual son aplicadas diferencialmente a varones y mujeres las categorías salariales del convenio colectivo. Específicamente, se refirió

10 Artículo $39^{\circ}$ "Igual trabajo, igual salario: Las condiciones a la que se refiere la presente convención Colectiva de trabajo, serán gozadas por el personal (ambos sexos), de cada establecimiento, debiéndose pagar al personal femenino, igual remuneración que al masculino, en caso de realizar igual trabajo" (CCT № 564/09 vigente para todo el personal encuadrado en FESTIQyPRA). 
a un plus salarial que reciben algunos operarios con mayor antigüedad por entrenar a nuevos trabajadores:

"Lo que pasa es por el tema del convenio, hay cosas que no están bien especificadas. Depende cómo se interprete. Porque de hecho tenemos gente, compañeras de años, y los muchachos tienen un porcentaje por ser capacitadores, por enseñar a una compañera cuando es nueva, sin embargo las chicas más antiguas cuando enseñamos a chicas más nuevas no hay esa diferencia" (operaria y delegada de 36 años. San Martín, mayo de 2011).

Hasta aquí, el lugar de las mujeres en las líneas se explicó, desde la perspectiva de las operarias, por las habilidades "naturalmente" femeninas o por las ambigüedades del convenio colectivo. En algún momento, una operaria para explicarme cómo funcionaba la máquina con la que trabajaba, hizo lo que toda maestra de escuela sabe que debe hacerse: la comparó con un objeto cotidiano que seguramente yo conocía y utilizaba en mi casa:

"En la máquina de Siano el enroscado es... vos fraccionas y tenés un roscador como si fuera así como... ¿viste el minipimer? Para que te des una idea" (operaria de 39 años. San Martín, marzo de 2011).

Una investigación sobre las ramas de la química, metalúrgica y del vestuario en la Región Metropolitana de São Paulo, Brasil, durante la década de los noventa (García, 2005), concluye que tareas que exigen minucia, disciplina o rapidez requieren habilidades adquiridas en el ámbito doméstico que no son valoradas socialmente y, en consecuencia, son vistas como predicados naturales del sexo femenino. Algo muy similar se constató entre las operarias químicas del Gran Buenos Aires.

Como afirma Kergoat (2003), los dominantes de la relación social de sexo no luchan contra las mujeres en una "guerra de sexos", sino que dirigen, desplazan y modulan sin cesar esa relación mediante la división sexual del trabajo. Esto puede observarse con mucha nitidez en el caso de la calificación. La calificación, concepto fundamental de la sociología del trabajo, cuando es interpelada desde la perspectiva de la relación social de sexo muestra su opacidad al poner de manifiesto que tanto su construcción individual como colectiva procede de manera completamente distinta en el caso de los hombres o de las mujeres; y que no puede ser entendida sin que se vuelva subjetiva.

Para Kergoat el problema reside en que tradicionalmente el concepto de trabajo ha sido pensado de manera coextensiva al de virilidad. De hecho, en el grupo de los hombres, no existe una interrupción de la continuidad entre trabajo/calificación/virilidad, mientras que para las mujeres autodefinirse o hacerse ver como calificadas es un proceso mucho más complejo, ya que las calificaciones ejercidas por las mujeres rara vez son reconocidas como tales. Esto así debido a que la calificación se funda en el valor socialmente atribuido a un trabajo. Pero además al hecho de que un trabajo es considerado más calificado entre más resulte una adquisición y menos un efecto de capacidades "naturales". 
Ahora bien, Kergoat encuentra que cualidades denominadas"naturales"son distintas de acuerdo con el sexo: las consideradas masculinas (la agresividad, la fuerza física, la voluntad de poder) son más valoradas socialmente que las consideradas femeninas (capacidad para relacionarse,"instinto"materno, la abnegación, la minuciosidad). De este modo, concluye, el proceso de construcción de la calificación varía de acuerdo con la posición que asuma en la relación social de sexos. Mientras que la calificación masculina individual o colectiva se construye socialmente, las cualidades femeninas se adquieren mediante un aprendizaje que erróneamente es vivido como individual por el hecho de que se efectúa en la esfera de lo privado y por esa razón no es valorado socialmente. De ahí que se establezca con los empleadores una relación de fuerzas desfavorable para las mujeres, y es sabido que de esa relación de fuerzas resulta la calificación.

Lo interesante de esta perspectiva es que permite la redefinición del concepto de calificación que habitualmente se aplica de modo concluyente para referirse a los puestos "femeninos" taylorizados como de baja o nula calificación. Se trataría, en cambio, de una calificación omitida, conformada por un cúmulo de saberes adquiridos en una socialización sexuada, fundamentalmente en el ámbito doméstico que, puestos en juego en el proceso de trabajo, son vistos como habilidades naturales (Pfefferkorn, 2007).

Según Castilla Ramos y Torres Góngora (2009), en el caso de las líneas de producción, las representaciones que moldean los principios de adaptación y eficacia de las obreras son inseparables de aquellas que gobiernan las tareas en el seno del hogar. Tanto en la casa como en la fábrica las mujeres utilizan sobre todo sus brazos, antebrazos, manos, así como los gestos de débil amplitud, pues las tareas son simples y repetitivas, por ello están en la cadena de montaje. Las actividades realizadas por las trabajadoras están intrínsecamente enlazadas con aquellas que desempeñan en sus hogares, esto se hace patente en las líneas de producción, en virtud de la naturaleza de las tareas: pese a la presencia de máquinas, fundamentalmente se ocupan de la inspección y el empaque.

¿En qué medida las destrezas productivas que despliegan las obreras químicas analizadas implican aquellos saberes y formas de hacer adquiridos en el ámbito doméstico familiar, como el trabajo doméstico y cuidado? Con la información recabada en esta investigación no ha sido posible responder a esta pregunta ${ }^{11}$. Su formulación, en cambio, plantea un desafío metodológico relativo a instrumentos de recolección de la información que permitan hacer comparables los procesos de trabajo en los establecimientos fabriles con las tareas de reproducción doméstica y familiar que se realizan cotidianamente con el propósito de sostener la vida. En este sentido, la utilización de entrevistas en profundidad posibilitó la reconstrucción parcial del proceso de trabajo en las líneas de montaje, pero resultó ser una técnica inadecuada para indagar

11 En otro trabajo (Anigstein, 2012) se han indagado las trayectorias familiares y las estrategias individuales y colectivas de organización del cuidado de las operarias fabriles. La elaboración de relatos etnográficos habilitó un conjunto de reflexiones en torno a la relación existente entre el modelo de organización del cuidado prescripto en las regulaciones laborales y las formas de participación y representación sindical de las mujeres. Sin embargo, este abordaje resultó insuficiente para comparar los procesos de trabajo en ambas esferas de la experiencia vital de las trabajadoras. 
el conjunto de actividades que involucra la producción del vivir, justamente por su carácter opaco, naturalizado e invisible.

\section{REFLEXIONES FINALES}

A lo largo de este escrito reflexionamos sobre dos problemas conceptuales. Por un lado, nos interesamos en el carácter ineludiblemente sexuado que asumen los procesos de trabajo en la producción capitalista. Por el otro, destacamos la centralidad del trabajo para comprender la opresión de género y la posición subalterna de las mujeres al interior del colectivo laboral asalariado.

La perspectiva de la relación social de sexos y la división sexual del trabajo provista por las investigadoras francesas Danielle Kergoat y Helena Hirata permitió reflexionar sobre la subalternidad de las mujeres ponderando al trabajo como categoría medular del análisis social y preconizando el carácter profundamente asimétrico de las relaciones entre el capital y el trabajo en las economías capitalistas. Junto a un conjunto de investigaciones realizadas en América Latina que comparten, en trazos gruesos, similar perspectiva analítica, y en diálogo con aportes de la sociología laboral y el marxismo clásico; el enfoque de la relación social de sexos habilitó, además, la comprensión de la construcción de la no calificación femenina en la fábrica y su relación con la división sexual del trabajo. De este modo, identificamos uno de los múltiples registros en los que se inscribe la intersección entre dominación de clase y opresión de género.

Nos detuvimos en la cuestión de la calificación femenina y el trabajo en líneas de montaje. Los procesos de trabajo en la fábrica seleccionada fueron descritos atendiendo a los mecanismos de control y disciplinamiento laboral aplicados, la organización de las tareas y las jornadas, entre otros.

A partir de esta descripción, afirmamos que las operarias son destinadas a las líneas de montaje donde realizan tareas repetitivas, que requieren gran velocidad, minuciosidad, motricidad fina y permanente concentración, siendo el embalaje y la inspección ocular sus principales funciones. Se halló que dichas tareas y los puestos de trabajo asociados a ellas son connotadas por las trabajadoras, sus pares varones y los empleadores como no calificados, femeninos y livianos (con relación a las tareas masculinas, que requieren mayor vigor físico o conocimientos técnicos reconocidos). Cuando estos puestos son ocupados por varones, mediante la adición de tareas auxiliares que suponen la puesta en juego de mayor esfuerzo físico, son recodificados como puestos masculinos. Esta redefinición de los puestos se objetiva en una recategorización, cuya consecuencia inmediata es un aumento en las remuneraciones percibidas.

Asimismo, se registró, en una proporción muy significativa, dolencias y enfermedades como consecuencia del tipo e intensidad del trabajo y la ocurrencia de accidentes laborales evitables. Y, con gran frecuencia de acuerdo con los testimonios recabados, conductas sumamente riesgosas -en su mayoría vinculadas a la presión de los empleadores, con el fin de aumentar la velocidad del trabajo-. 
Atendiendo a estas características del proceso de trabajo, analizamos cómo las destrezas productivas de las obreras guardan una estrecha relación con saberes adquiridos en el ámbito doméstico, invisibilizados y decodificados como habilidades naturales. En esta dirección, sugerimos que las operarias mantienen una relación ambivalente con la calificación, no problematizan la división sexual del trabajo en la planta y naturalizan sus destrezas. Sin embargo y conjuntamente, perciben inequidades entre sexos. Un ejemplo de ello es el plus salarial que no perciben las operarias por entrenamiento de nuevos trabajadores, otorgado a operarios varones exclusivamente.

\section{REFERENCIAS BIBLIOGRÁFICAS}

Abal Medina, Paula y Diana Menéndez, Nicolás (comp.) (2011): Colectivos Resistentes. Procesos de politización de trabajadores en la Argentina reciente. Buenos Aires. Editorial Imago Mundi.

Abal Medina, Paula; Arias, Cora; Battistini, Osvaldo; Busso, Mariana; Crivelli, Karina y Míguez, Pablo (2009): Senderos bifurcados. Prácticas sindicales en tiempos de precarización del trabajo. Buenos Aires. Prometeo Libros.

Alaluf, Mateo (2003):"La sociología del trabajo a través de una relectura de Claude Durand. Situación del trabajo, calificaciones, competencias y clase obrera", en Cuadernos de Relaciones Laborales 21, № 2, pp. 93-102. Madrid. Universidad Complutense de Madrid.

Anigstein, Cecilia (2012): Fabriqueras. Trabajo, familia y sindicalismo en la industria química y cosmética. Gran Buenos Aires, 2007-2011. Tesis de maestría del Programa de formación en Posgrado en Ciencias Sociales UNGS-IDES. Buenos Aires. Inédita.

Antúnez, Ricardo (2005): Los sentidos del trabajo. Ensayo sobre la afirmación y la negación del trabajo. Buenos Aires. Herramienta Ediciones - Taller de Estudios Laborales.

Arango, Luz Gabriela (1997): "La clase obrera tiene dos sexos. Avances de los estudios Latinoamericanos sobre género y trabajo", en Nómadas №6. Bogotá. Departamento de Investigaciones, Universidad Central-DIUC.

Battistini, Osvaldo (Compilador) (2004): El trabajo frente al espejo. Continuidades y rupturas en los procesos de construcción identitaria de los trabajadores. Buenos Aires. Prometeo Libros.

Carneiro Araújo, Ângela Maria (2005): "Gênero nos studos do trabalho", em Maria Lygia Quartim de Moraes org. Gênero na fronteira do Sul. 1ª Ed., Campinas, Brasil. Pagu/ Unicamp.

Carneiro Araújo, Ângela Maria y Menicucci de Olivera, Eleonora (2006):"Reestruturação Produtiva e saúde no setor metalúrgico. a percepção das trabalhadoras", en Sociedade e Estado, Brasília, Brasil, v. 1, pp. 169-198, jan/abr. 2006. 
Carneiro Araújo, Ângela Maria; Aguiar Amorin, Elaine Regina; Clemente Ferreira, Verônica (2004): "O sentidos do trabalho da mulher no contexto da Reestruturação Produtiva", Artigo a ser apresentado no VIII Congresso Luso-Afro Brasileiro de Ciências Sociais, Coimbra, Portugal, 16 a 18 de setembro de 2004.

Castilla Ramos, Beatriz y Torres Góngora, Beatriz (2009): "Del hogar a la fábrica. Trabajadoras de las empresas transnacionales en Yucatán, México", en TRACE 55, junio 2009. México. Centro de Estudios Mexicanos y Centroamericanos, pp. 31-52.

Cifarelli, Viviana; Martínez, Oscar; Pérez Crespo, Guillermo (2007): De eso no se habla: organización y lucha en el lugar de trabajo. Cuadernos del TEL. Buenos Aires. TEL

Coriat, Benjamín (2008): El taller y el cronómetro. Ensayo sobre el taylorismo, el fordismo y la producción en masa. México. Siglo Veintiuno Editores.

De la O., María Eugenia (2006): "El trabajo de las mujeres en la industria maquiladora de México: Balance de cuatro décadas de estudio", en AIBR Revista de Antropología Iberoamericana, Ed. Electrónica, vol. 1, número 3, agosto-diciembre 2006, Madrid, pp. 404-427.

García Castro, Mary (1992):"O conceito de gênero e as análises sobre mulher e trabalho. notas sobre impasses teóricos". Cad. CRH, Salvador, 17, pp. 80-105.

Garza Toledo, Enrique (2001): "Problemas clásicos y actuales de la crisis del trabajo", en El trabajo del futuro y el futuro del trabajo, Neffa, de la Garza (Comp), FLACSO, Buenos Aires.

Gilly, Adolfo (1985): "La mano rebelde del trabajo", en Cuadernos del Sur, Número 1, enero-marzo de 1985. Buenos Aires. Editorial Tierra del Fuego.

Hartman, Heidi (1980): "Capitalismo, patriarcado y segregación de los empleos por sexo", en Z. R. Eisenstein comp., Patriarcado, capitalismo y feminismo socialista. México. Siglo Veintiuno Editores, pp. 186-220.

Harvey, David (1998): La condición de la posmodernidad. Investigación sobre los orígenes del cambio cultural. Amorrortu Editores, Avellaneda, provincia de Buenos Aires.

Hirata, Helena (2001): "Globalização e divisão sexual do trabalho", Cadernos Pagu 17/18 2001/02, pp. 139-156.

2010. “División sexual del trabajo. Puntos de método". Publicado en Aporrea el 31/10/2010. URL. http.//www.aporrea.org/ideologia/a98056.html

Hirata, Helena; Kergoat, Danielle (2007): "Novas configurações da divisão sexual do trabalho", en Cadernos de Pesquisa, v. 37, n. 132, São Paulo, set./dez. 2007, pp. 595-609. 
Hutchison, Elizabeth Quay (2005): Labores propias de su sexo: género y trabajo en Chile urbano, 1900-1930, trans. Jacqueline Garreaud. Santiago. LOM Ediciones.

Kaplan, Temma (1990): "Conciencia femenina y acción colectiva: el caso de Barcelona”, en James S. Amelang y Mary Nash, comp., Historia y Género: Las mujeres en la Europa Moderna y Contemporánia. Valéncia, Edicions Alfons el Magnànim, pp. 267-296.

Kergoat, Danielle. (2003): "De la relación social de sexo al sujeto sexuado", en Revista Mexicana de Sociología, año 65, núm. 4, octubre-diciembre 2003, México, pp. 841-861.

López Aspeitía, Luis (2010): "Identidades en la línea. Maquiladoras y figuras de la femineidad en la frontera norte de México", en Revista Mexicana de Sociología, vol. 72, No 4. México, oct./dic. 2010.

Martínez, Oscar (comp.) (1994): Pensando la Reconversión: Una Visión Crítica de la Flexibilidad y la Calidad Total. Buenos Aires. CIPES.

Marx, Karl (2002): El capital. El proceso de producción del capital. Tomo I, Vols. 1 y 2. Buenos Aires. Siglo Veintiuno Editores Argentina.

Pequeño Rodríguez, Consuelo (2005): "Consideraciones para el estudio del trabajo de las mujeres en la industria de la maquiladora", en NOESIS Revista de Ciencias Sociales y Humanidades, julio-diciembre, año/vol. 15, número 028. Universidad Autónoma de Ciudad Juarez, México, pp. 33-55.

Pfefferkorn, Roland (2007): "El trabajo. Un eje fundamental de las relaciones sociales de sexo", en Revista de Ciencias Sociales No 18, Departamento de Ciencias Sociales, Universidad Arturo Prat, lquique, invierno 2007, pp. 53-70.

Roldán, Martha (1992): “Un debate pendiente: Reconversión industrial, desregulación y nuevos procesos de trabajo flexibles en el contexto latinoamericano de los 90. ¿Hacia una perspectiva de género?", en Bustos, T. y Palacio, G. (comp.) El trabajo femenino en América Latina. Los debates de la década de los noventa. México DF. Eds. Universidad de Guadalajara, ILSA.

Scott, Joan W. (1993): "La mujer trabajadora en el siglo XIX", en Historia de las mujeres, Duby y Perrot Eds., tomo 5, “El siglo XIX”, Fraisse y Perrot comp., pp. 405-435.

Stroobants, Marcelle (1999): "Trabajo y competencias. Recapitulación crítica de los enfoques sobre los saberes en el trabajo", en Calificaciones y Empleos No 21, 10 Trimestre de 1999. Buenos Aires. Piette/Céreq.

Wyczykier, Gabriela (2009): De la dependencia a la autogestión laboral: Sobre la reconstrucción de experiencias colectivas de trabajo en la Argentina contemporánea, Buenos Aires. Prometeo/UNGS. 